\title{
A divergent insect-specific flavivirus that causes overt cytopathic effect in a mosquito cell-line
}

D. Duque Ferreira*, A.P. Alves de Matos**, A. Esteves*, A. Lopes*, A.P. Almeida***, J. Piedade* and R. Parreira*

*Unidade de Microbiologia Médica (Grupo de Virologia)/Unidade de Parasitologia e Microbiologia Médicas (UPMM), Instituto de Higiene e Medicina Tropical (IHMT), Universidade Nova de Lisboa (UNL), Rua da Junqueira 100, 1349-008 Lisboa, Portugal

**Anatomia Patológica, Centro Hospitalar de Lisboa Central - HCC, Rua da Beneficência 8, 1069-166 Lisboa, Portugal; Centro de Estudos do Ambiente e do Mar (CESAM/FCUL) - Faculdade de Ciências da Universidade de Lisboa and Centro de Investigação Interdisciplinar Egas Moniz (CiiEM), Quinta da Granja , Monte de Caparica, 2829-511 Caparica, Portugal

***Unidade de Parasitologia Médica/UPMM, IHMT/UNL, Rua da Junqueira 100, 1349-008 Lisboa, Portugal

The genus Flavivirus (Flaviviridae) includes over seven dozen known enveloped viruses with ssRNA genomes, the evolution, geographic dispersion and epidemiology of which seems to have been shaped both by environmental constraints (involving ecological factors and human activities), and by their replication in vertebrate and invertebrate hosts. Most bona-fide flaviviruses have been classified as mosquito or tick-borne viruses, although for some no known invertebrate vector has yet been found. Over time, a divergent cluster including viral agents that seem to be specific of insect (Insect-Specific Flaviviruses-ISF) has been associated with the genus. The classification of ISF as flaviviruses is supported by their genomic organization, protein hydropathy plots, conserved polyprotein cleavage sites and enzyme domains, which are similar to those of classical flaviviruses. However, they are antigenically distinct, and share approximately the same level of nucleotide sequence identity with other members of the genus, as when compared to the members of the two other genera in the Flaviviridae family $[1,2]$.

This work reports the initial characterization, including near full-length sequence analysis, of an ISF which seems to form a divergent lineage within the ISF radiation (Figure 1), as indicated by phylogenetic analysis based on ac multiple sequence alignment of the NS5 flavivirus coding sequence. This virus, tentatively designated OCFV (Ochlerotatus caspius flavivirus) was isolated from clarified macerates of a pool of Aedes (Ochlerotatus) caspius (Pallas, 1771) adult mosquitoes (lab code number 174) using the Ae. albopictus-derived C6/36 cell line. The mosquitoes from which OCFV was isolated were collected using CDC-traps baited with $\mathrm{CO}_{2}$, and are amongst those found in high densities in the coastal, and estuarine, districts of Setúbal and Faro [3]. Detection of viral genomes by RT-PCR using RNA directly extracted from supernatants of C6/36 infected cultures, collected $24 \mathrm{~h}$ post-infection, indicated that the virus replicates rapidly in these cells. Nevertheless, but as expected for an ISF, it does not replicate in Vero cells. Unexpectedly, unlike most ISF OCFV seems to cause overt cytopathic effect in C6/36 cells, which rapidly detach from a solid support and round-up soon (24-48h) after infection. Electronmicroscope analysis of thin-sections of $\mathrm{C} 6 / 36$ cells at $48 \mathrm{~h}$ post-infection with OCFV revealed nuclear hyperplasy, and an evident enlargement of the intracisternal space of the nuclear envelope, which is filled by multiple sized vesicles. In some cells, a complex network of apparent membrane trabeculae was also clearly evident (Figure 2A). Viral particles seem to assemble at the endoplasmatic reticulum, from which viruses reach the cell surface by vesicular transport (Figure 2B). Unexpectedly for a flavivirus, but as previously observed [4-6], viral particles also seem to gemulate directly from the cytoplasmic membrane (Figure 2C).

References

1. Cook S. and Holmes E.C., Arch. Virol., 151:309-325, 2006.

2. Gould E.A. et al., Adv. Virus. Res., 59:277-314, 2003,

3. Almeida A.P., et al., Trans. R. Soc. Trop. Med. Hyg., 102:823-832, 2008.

4. Hase T. et al., Arch. Virol., 92:273-291, 1987.

5. Ng M.L. et al., Arch Virol., 137:303-313, 1994.

6. Ng M.L. et al., J. Med. Virol., 65:758-764, 2001. 


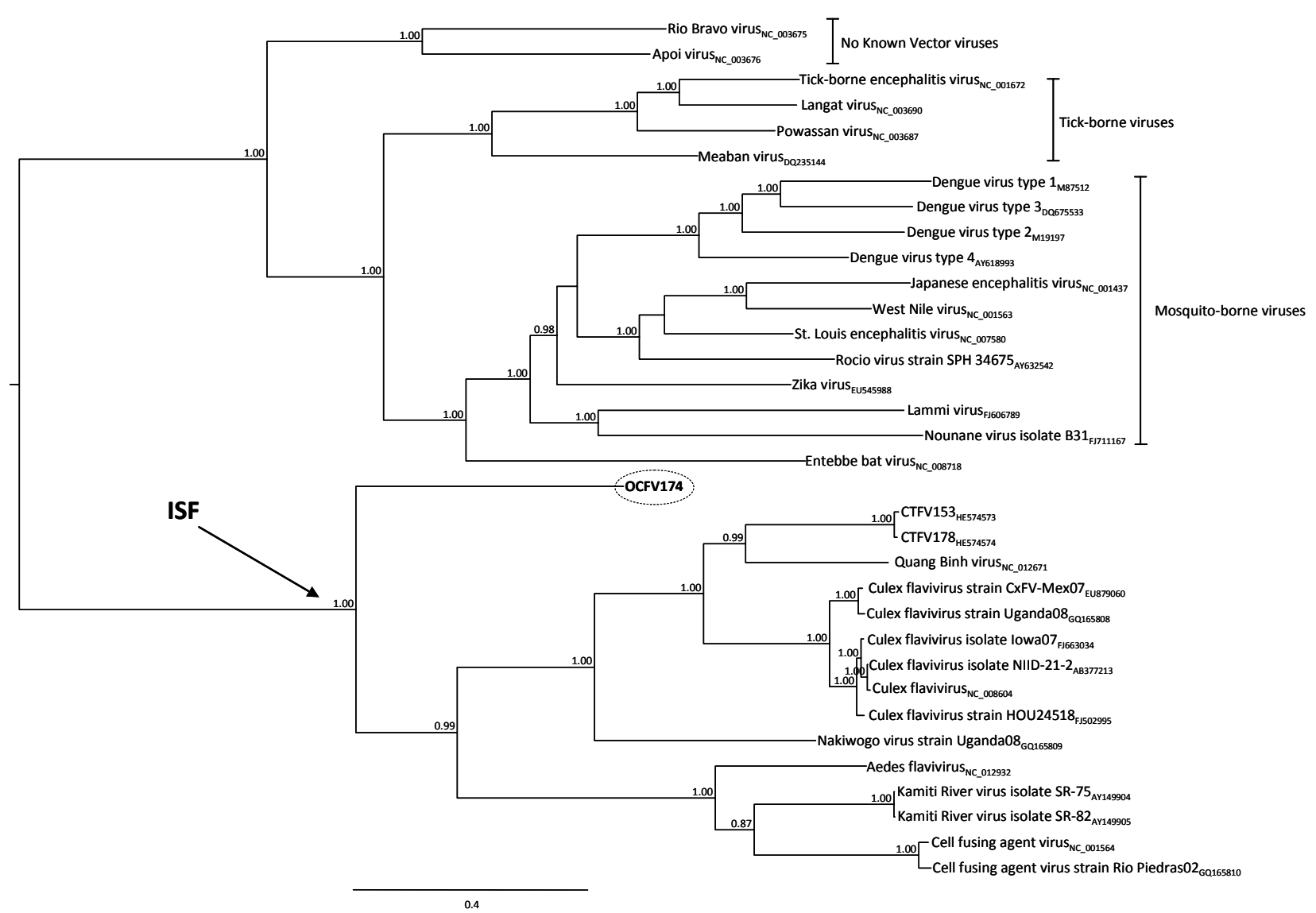

Figure 1. Phylogenetic analysis (Bayesian approach) of OCFV (clone 174, interrupt line) with other flaviviruses (mosquito-borne, tick-borne and no known vector viruses) based on the analysis of NS5 (viral polymerase) nucleotide sequences. The ISF radiation, where OCFV forms a divergent lineage, is indicated by the arrow.

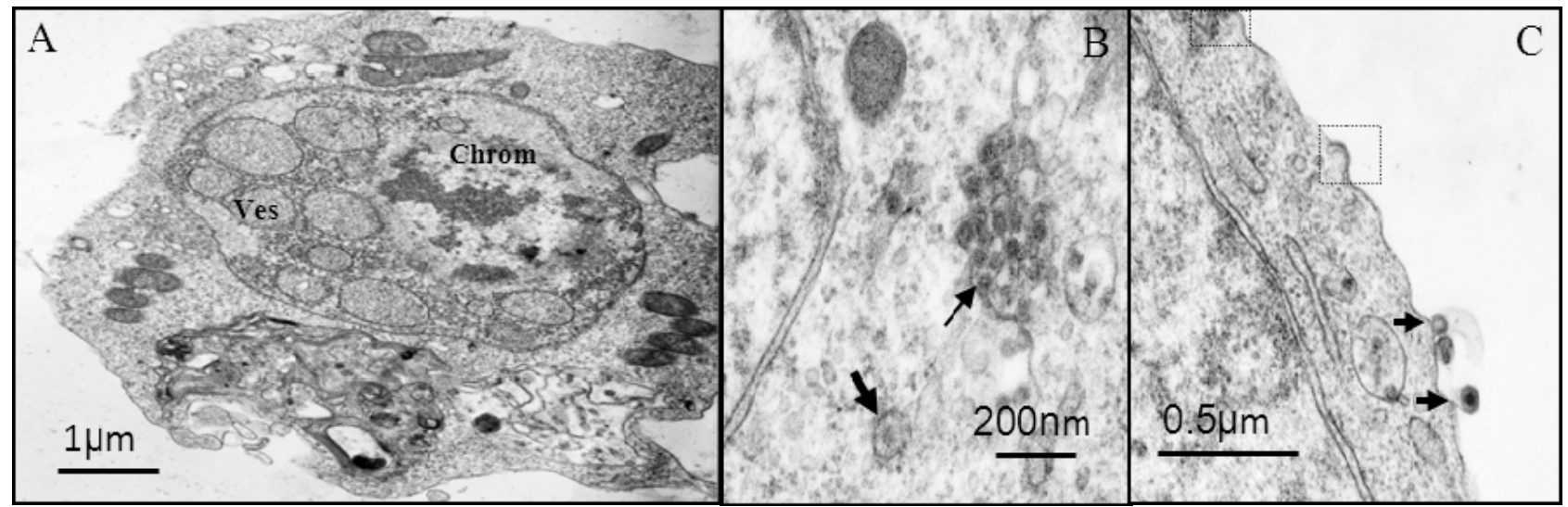

Figure 2. TEM micrographs of C6/36 cells at $48 \mathrm{~h}$ post infection with OCFV strain 174 . Infected cells show nuclear hyperplasy and accumulation of vesicles and trabeculae in the intercisternae space of the nuclear envelope (A). Viral particles accumulate in the endoplasmic reticulum as well as can be identified in cytoplasmic vesicles (arrows in B and C). Assembly of viral particles at the cytoplasmic membrane is also suggested (interrupt line in C). Mature virions were observed at the cell surface (thick arrow in C). 